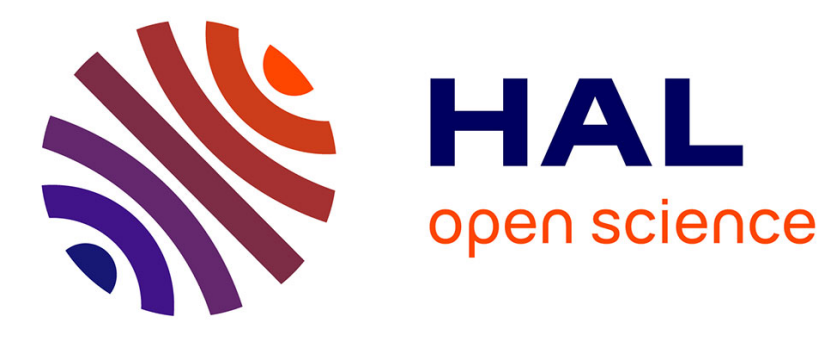

\title{
Trois propositions pour fonder une entreprise convivialiste
}

\author{
Armand Hatchuel, Blanche Segrestin
}

\section{To cite this version:}

Armand Hatchuel, Blanche Segrestin. Trois propositions pour fonder une entreprise convivialiste. Revue du MAUSS, 2016, S'émanciper, oui, mais de quoi ?, 48 (second semestre). halshs-01394413

\section{HAL Id: halshs-01394413 https://shs.hal.science/halshs-01394413}

Submitted on 9 Nov 2016

HAL is a multi-disciplinary open access archive for the deposit and dissemination of scientific research documents, whether they are published or not. The documents may come from teaching and research institutions in France or abroad, or from public or private research centers.
L'archive ouverte pluridisciplinaire HAL, est destinée au dépôt et à la diffusion de documents scientifiques de niveau recherche, publiés ou non, émanant des établissements d'enseignement et de recherche français ou étrangers, des laboratoires publics ou privés. 


\title{
Trois propositions pour fonder une entreprise convivialiste
}

\author{
Armand Hatchuel et Blanche Segrestin
}

\section{LE DIVORCE ENTRE GRANDES ENTREPRISES ET SOCIETES}

Depuis trois décennies, la rentabilité financière promise aux actionnaires est devenue l'impératif de gestion des grandes entreprises cotées. Au lieu d'investir, il est devenu courant d'utiliser une part importante des profits pour soutenir les cours de bourse par des rachats massifs de titres. L'investissement ou la fiscalité en ont notamment été affectés, ce qui a érodé l'emploi, les salaires et les ressources publiques. Ce sont donc la justice sociale (explosion des inégalités de patrimoine et de revenus) et la prospérité générale qui ont été durement atteintes par la mutation du mode de gouvernement des grandes entreprises, au risque de mettre en danger l'avenir des entreprises elles-mêmes.

Simultanément, États, associations et organismes internationaux ont demandé aux chefs d'entreprise d'être «socialement et environnementalement» responsables. Mais le voudraient-ils qu'ils ne le pourraient pas vraiment : ce serait agir contre les règles dominantes de la Corporate Governance (gouvernement des sociétés) qui les somment d'agir au nom et dans le seul intérêt des actionnaires. Incités financièrement par des rémunérations exorbitantes indexées sur la valeur des actions, menacés de révocation s'ils ne fournissent pas des dividendes satisfaisants, les dirigeants les plus éclairés n'ont en réalité guère de latitude pour défendre une stratégie qui ne serait pas celle de la «valeur actionnariale». Ils ne peuvent ni revendiquer une quelconque loyauté à un territoire national - on l'a bien vu avec les stratégies d'optimisation fiscale - ni rechercher une relative équité vis-à-vis des intérêts des personnels ou des partenaires de l'entreprise.

Il en résulte un fait majeur et inédit dans l'histoire des sociétés occidentales: le succès des grandes entreprises n'est plus mécaniquement source de progrès collectifs et de création d'emplois. Ce divorce est d'autant plus déstabilisateur que ces entreprises consomment le gros des ressources intellectuelles, humaines et publiques d'une nation. Il signale aussi l'obsolescence du savoir économique et social classique qui n'a pas mesuré, depuis un siècle, les conséquences phénoménologiques et épistémologiques de la formation des grandes entreprises dans l'Histoire occidentale.

Il nous a donc semblé que le projet convivialiste ne peut se désintéresser de la dérive financière de la grande entreprise. Il ne peut non plus s'en remettre, pour la corriger, à un « renversement du capitalisme » dont on ne sait quelle signification opératoire lui prêter.

Dans cet article, nous ferons trois propositions pour une refondation de l'entreprise. Elles ne prétendent pas répondre à tous les problèmes que pose la dérive de la grande entreprise, mais elles abordent des questions fondamentales tout en restant opératoires et réalisables à moyen terme. Bien qu'elles aient été développées indépendamment du projet convivialiste, ces propositions nous semblent aussi cohérentes avec les principes et les valeurs de ce mouvement ${ }^{1}$.

\section{PROPOSITION 1 : RECONNAITRE L'ENTREPRISE COMME UN PROJET DE CREATION COLLECTIVE QUI NE SE CONFOND PAS AVEC LA SOCIETE DE CAPITAUX}

L'« entreprise » n'est ni le marchand ni l'entrepreneur de la tradition. La notion renvoie à des formes de collectifs apparus relativement récemment. Nés d'abord comme « grandes industries » avant de s'étendre à tous les types d'activité, ces collectifs se sont distingués par leur capacité, sans précédent historique, à produire des biens et des services novateurs, à coordonner des taches multiples et complexes et à créer des emplois fondés sur la qualification et l'acquisition de compétences. La «grande entreprise moderne» est donc née à la fin du $\mathrm{XIX}^{\mathrm{e}}$ siècle, bien après les sociétés de capitaux qui servaient depuis longtemps d'instrument financier au capitalisme. Portée par les nouvelles forces sociales que furent les ingénieurs, les techniciens et les cadres, elle a

\footnotetext{
${ }^{1}$ Ces propositions sont issues de plusieurs travaux conduits à Mines ParisTech, PSL Research University, et en liaison avec plusieurs autres chercheurs dans le cadre du Collège des Bernardins : "Entreprises : formes de propriété et responsabilités sociales ». Pour des développements plus approfondis, on pourra se reporter aux ouvrages: Segrestin et Hatchuel [2012] ; Favereau et Baudouin [2015] ; Segrestin, Levillain, Vernac et Hatchuel [2015].
} 
été pensée, dans le sillage des Lumières et du développement scientifique, comme un projet de construction technique et sociale.

Tout au long du $\mathrm{XX}^{\mathrm{e}}$ siècle, les pouvoirs publics et le droit ont tenté d'encadrer les conflits créés par la nouvelle «entreprise moderne » au travers de diverses branches législatives qui se sont développées en restant quasiment étanches les unes aux autres (droit du travail, droit commercial, droit de la propriété intellectuelle). Ainsi, le droit organise les sociétés commerciales (par exemple, les sociétés anonymes) et leur gouvernance, sans référence à d'autres composantes de l'entreprise, en dehors des associés, et en excluant les salariés des décisions aussi critiques que la nomination ou la révocation des dirigeants.

In fine, l'entreprise n'a jamais été pensée en tant que telle. Durant les deux premiers tiers du $\mathrm{XX}^{\mathrm{e}}$ siècle, les entreprises se sont développées malgré ce vide comme une composante centrale du développement des États et des nations. Mais on réalise, aujourd'hui, que ce vide juridique a laissé la porte ouverte aux doctrines économiques et financières les plus dangereuses. Ainsi, l'amalgame entre entreprise et société de capitaux a fini par se retourner contre l'entreprise.

Il est donc crucial que l'entreprise soit refondée en respectant ce qu'elle est sous tous ses aspects, c'est-à-dire un sujet collectif créateur, irréductible à la seule recherche du profit, et pleinement responsable comme tout autre collectif. Cela devrait passer, de notre point de vue, par la proposition préalable suivante :

Reconnaître la singularité de l'entreprise comme un collectif de création collective et affirmer que l'individuation de ces collectifs construit la société à mesure qu'ils se construisent eux-mêmes.

Cette proposition a d'importantes implications, qui font écho aux principes convivialistes :

- les entreprises ne se contentent pas de produire des richesses déjà connues, elles participent à transformer la définition des richesses. Elles ont donc la responsabilité de la création (destruction) de nouveaux communs techniques, humains et sociaux. Elles ne sont pas de simples agents économiques, mais des collectifs de régénération du monde commun;

- à l'instar de tout collectif, leurs fonctionnements doivent être aussi fondés sur l'idée d'opposition maîtrisée et créatrice.

Cette fondation générale conduit à deux propositions à vocation opératoire et réformatrice, l'une est relative à un nouveau contrat de société, l'autre à un nouveau statut du dirigeant d'entreprise.

\section{PROPOSITION 2 : CREER UNE NOUVELLE SOCIETE A « OBJET SOCIAL ETENDU »}

La définition de l'entreprise que nous venons d'élaborer conduit à une révision majeure de la vulgate économique : on ne peut réduire l'objectif d'une entreprise au seul profit! Certes, dans un monde où les échanges sont monétarisés, le profit est une condition nécessaire d'équilibre et de pérennisation. Mais il ne saurait déterminer, à lui seul, la nature de l'entreprise et la logique de tous ses choix.

En effet, si l'entreprise participe à la création de nouveaux communs techniques, humains et sociaux, le seul critère du profit est insuffisant, voire trompeur, pour juger de sa contribution à la société ou au territoire qui l'accueille! Il faut donc corriger un postulat ancien du droit, exprimé dans les articles 1832 et 1833 du code civil, selon lequel la création d'une société a pour seul but le partage des pertes et des bénéfices entre des associés ! Une société ne peut donc refléter la réalité d'une entreprise qu'en définissant, au-delà des bénéfices, la mission quelle s'assigne. Mission exprimant les engagements que cette société prend vis-à-vis de tous ceux avec lesquels elle veut construire de nouveaux communs.

Exprimés initialement par les associés, ces engagements s'étendraient évidemment à tous ceux qui travaillent dans l'entreprise mais aussi à tous ceux qui accepteraient de partager ces engagements. Pour incarner juridiquement cette mission en droit des sociétés, nous proposons que soit créée une norme d'entreprise fondée sur une société à objet social étendu.

Cette norme instaurerait un collectif distinct des formes existantes (sociétés, association, GIE, etc.). Elle se démarquerait d'abord d'une société commerciale classique par l'insertion dans son objet social d'objectifs scientifiques, économiques, environnementaux, humanitaires et sociaux, «étendus ». Jusqu'à présent, l'objet social fait référence au domaine d'activité de l'entreprise. Si une nouvelle norme autorisait des objets plus ouverts, alors les associés pourraient s'engager sur de nouveaux objectifs allant au-delà de leur intérêt économique (comme le bien-être des salariés ou la volonté de minimiser les effets des activités sur l'environnement, etc.). Les actionnaires restaureraient ainsi l'étendue et la latitude de gestion des dirigeants tout en affirmant les valeurs de leur entreprise. De telles normes de sociétés existent déjà aux États-Unis. Mais il reste à construire ce type de société dans le cadre du droit français et européen. Une telle loi permettrait de créer des organisations hybrides entre associations à but non lucratif et entreprises. En invitant les sociétés à définir 
statutairement leur mission - et donc les conditions générales de leur responsabilisation au regard de la vie sociale -, on corrige une des contradictions anthropologiques fondamentales des sociétés modernes.

En effet, en justifiant l'existence de collectifs de vie et de travail, dont le seul but est la maximisation du profit, on a organisé une contradiction majeure. On a accepté que, dans le cadre même de la vie sociale commune, il existe des entités, en outre particulièrement influentes et puissantes, qui n'ont pas pour objet de participer à cette vie sociale commune! Ces entités seraient ainsi exonérées de toute responsabilité dans la construction de la société qui les accueille et leur permet d'exister. La thèse libérale objecterait à cette affirmation que ces entités ont des responsabilités, strictement définies par le droit. Mais le droit et la théorie politique modernes se sont historiquement construits au début du XIX ${ }^{\mathrm{e}}$ siècle dans un monde sans grandes entreprises, sans recherche scientifique et sans cadre théorique permettant de penser la création (et la destruction) de communs techniques, humains et sociaux ${ }^{2}$. Le droit a donc pu postuler l'absence de responsabilité des actionnaires, y compris les plus puissants et les plus influents d'entre eux, négligeant ainsi les décisions qu'ils ont contribué à faire prendre, qu'ils ont formellement approuvées, ou leur implication directe dans la nomination des dirigeants de leur société. On se souvient que lorsque l'accident d'un puits de British Petroleum avait durablement pollué la Louisiane, le gouvernement américain n'avait pas pu, à titre conservatoire, bloquer la distribution de dividendes aux actionnaires de l'entreprise.

L'histoire moderne a donc organisé une double irresponsabilité: elle a légitimé le fait qu'une société/entreprise ne poursuive que des buts lucratifs et elle a fondé l'irresponsabilité des associés pour les actions de l'entreprise. La création de la société à objet social étendu autorise à prendre une direction inverse : elle substitue à cette double irresponsabilité un cadre de mission qui engage l'entreprise et ses actionnaires au devoir commun : prendre sa part dans le progrès collectif.

\section{PROPOSITION 3 : DEFINIR LE DIRIGEANT DE GRANDE ENTREPRISE COMME AUTORITE HABILITEE A GERER AU NOM DU BIEN COMMUN ET RESPONSABLE DE LA SOLIDARITE EQUITABLE ENTRE LES PARTIES DE L'ENTREPRISE}

Il est trop souvent admis que les dirigeants tirent leur pouvoir d'une délégation permise par un droit de propriété : celui des actionnaires. Pourtant, l'irresponsabilité des actionnaires est incompatible avec une telle délégation. En réalité, le droit accorde au chef d'entreprise des pouvoirs et des responsabilités sans rapport avec le mandat des actionnaires. Ainsi, les décisions du chef d'entreprise engagent des ressources, des risques et des potentiels sociaux qui vont bien au-delà des capitaux apportés par les actionnaires. Les décisions du chef d'entreprise pèsent sur les capacités et la vie des salariés, sur les fournisseurs qui lui ont fait confiance, sur la vie des territoires qui l'accueillent. Le pouvoir et la responsabilité des dirigeants d'entreprise ne peut donc découler des seuls associés qu'ils sont censés représenter.

Il nous faut prendre conscience que la modernité a construit une forme d'autorité majeure, mais sans penser ni la nature de cette autorité ni l'ensemble des responsabilités, autres que comptables, qui doivent lui être assignées ${ }^{3}$ indépendamment du mandat des associés.

Nous proposons de penser le chef d'entreprise comme une autorité habilitée ${ }^{4}$ par l'État et à laquelle est confiée la responsabilité de conduire l'entreprise dans le sens d'un intérêt général associé au progrès des sociétés nationales et civiles qui l'accueille et des partenaires qu'il mobilise. On peut penser ici au capitaine de bateau, figure particulièrement étudiée et encadrée dans les législations les plus anciennes, ainsi qu'aux figures plus modernes des commandants de bord de l'aviation, ou mêmes aux professions libérales assujetties à des responsabilités spécifiques vis-à-vis de l'État et des individus.

Ces figures d'autorité ont toujours été construites par une double légitimité : celle du mandat local qu'un mandataire (armateur, compagnie, client) leur confie, et celle de la société qui les habilite à défendre des intérêts supérieurs à ceux de ce mandataire : par exemple, pour les commandants de bord, celui de la sécurité des personnes à bord ou de toutes celles qui pourraient être affectées (au sol notamment) par ses décisions.

Une telle habilitation signifierait que le chef d'entreprise a le devoir d'assurer un principe de solidarité et d'équité entre les intérêts divers que ses décisions vont affecter, dans et hors de l'entreprise. Là aussi, l'histoire de la figure du capitaine de bateau est riche d'enseignements ${ }^{5}$. En effet, un principe de solidarité est connu

\footnotetext{
${ }^{2}$ Pour une discussion plus approfondie de ce point, on pourra se reporter à Levillain, Segrestin et Hatchuel [2014].

${ }^{3}$ À l'exception de l'Allemagne de l'après-guerre qui a adopté la codétermination des entreprises. L'un des fondements de ce modèle est que l'autorité des dirigeants ne peut provenir des seuls associés et doit aussi recevoir l'assentiment des salariés.

${ }^{4}$ Nous suivons ici l'argumentation et la proposition de Stéphane Vernac.

${ }^{5}$ Voir Segrestin et Hatchuel [2011].
} 
depuis l'Antiquité sous le nom de « règle des avaries communes » : le capitaine d'un navire a le droit de jeter des cargaisons à la mer si ce geste assure le salut de l'expédition. Le capitaine est libre de son choix, mais la perte consentie ne retombe pas uniquement sur le propriétaire de la cargaison sacrifiée : elle doit être partagée, d'après la loi internationale, entre tous ceux qui en bénéficient, c'est-à-dire tous ceux dont le bien a été sauvé par ce sacrifice « commun ».

À l'instar des avaries communes, le chef d'entreprise doit être responsable des intérêts supérieurs qui lui incombent et de la juste solidarité entre les parties de l'entreprise quant aux effets de sa gestion. On peut lire, dans ce principe d'habilitation et de solidarité, un concept authentiquement convivialiste.

\section{Références citées}

FAVEREAu Olivier, BAudouin Roger (dir), 2015, Penser l'entreprise. Nouvel horizon du politique, Collège des Bernardins/Humanités, « Perspectives et propositions », Paris.

LeVILlain Kevin, Segrestin Blanche, Hatchuel Armand, 2014, « Repenser les finalités de l'entreprise. La contribution des sciences de gestion dans un monde post-hégélien », Revue française de gestion, $\mathrm{n}^{\circ} 245$ (8).

SEGRESTIN Blanche, HATCHUEL Armand, 2012, Refonder l'entreprise, Seuil, «La République des Idées », Paris.

- 2011, «Autorité de gestion et avaries communes : pour un complément du droit de l'entreprise ?», Finance Contrôle Stratégie, vol. 14, n 2, juin, p. 9-36.

Segrestin Blanche, LeVIllain Kevin, Vernac Stéphane, HATchuel Armand (dir), 2015, La Société à Objet Social Etendu : un nouveau statut pour l'entreprise, Presses des Mines, «Économie Gestion », Paris. 